rauchen doch bereits 40\% dieser Altersgruppe, so dass der Wettbewerb sich um das Vermeiden einer Abhängigkeit von Raucherwaren bemüht. Zur künftig besseren Koordination von Aktivitäten im Jugendbereich wird ein Ausschuss geschaffen.

Seitens der FMH benutzen wir die Gelegenheit, Sie kurz über die Tagung des «Tobacco Control Resource Centre» (TCRC; begründet durch das «European Forum of Medical Associations" und der WHO, unterstützt durch das Programm Europa gegen Krebs des Europarats und der British Medical Association) vom März $2000 \mathrm{zu}$ orientieren. Das TCRC hat zum Ziel, eine europaweite Informationszentrale zu sein, Materialien speziell für die Ärzteschaft in Europa zu publizieren, europaweit eine Studie zu den Rauchgewohnheiten der Ärzteschaft zu koordinieren sowie Weiter- und Fortbildungen anzubieten. Rechtzeitig für die Tagung vom März 2000 konnte die Monographie "Ärzte und Tabak; die grosse medizinische Herausforderung" herausgebracht werden. Sie existiert in deutscher, französischer, italienischer, spanischer, englischer und portugiesischer Version. Bei unserer Abteilung können Exemplare in unseren drei Landessprachen gegen Einsenden eines an Sie selber adressierten und frankierten A4 Briefumschlags bestellt werden. (Es sind darin Kapitel über besondere Risikogruppen, über Rolle der Ärzte und Steigerung des ärztlichen Bewusstseins bezüglich des Eindämmens des Rauchens, Strategien zur Tabakkontrolle sowie Angaben zu einem Verhaltenskodex zu von der Tabakindustrie finanzierten Forschungen zu finden.) Drei Leitmotive wurden für die nächsten Jahre verabschiedet:

1. Die europäischen nationalen Ärztegesellschaften sollten sich bereit erklären, alle drei bis fünf Jahre eine Erhebung über die Rauchgewohnheiten ihrer Mitglieder durchzuführen.

2. Bezüglich des Rauchstopps sollen die vier A im Visier behalten werden: "Ask patients about smoking - Advise on stopping smoking - Assist those who are ready to quit - Arrange a followup visit."

3. Sollen sich die nationalen Ärztegesellschaften vermehrt um restriktive Gesetzgebungen in ihrem Land bemühen. ( $\mathrm{Zu}$ denken ist etwa an die Erhöhung des Preises für Tabakwaren, an die Einschränkung der Reklame, an das Anbieten kleinerer Packungseinheiten, das offenbar in Holland bereits zu guten Erfolgen bei Jugendlichen geführt hat.)

Es ist übrigens erwähnenswert, dass die «Food and Drug Administration" versucht, Nikotin als Droge und Tabakprodukte als drogenliefernde Artikel zu regulieren. Soeben wird bekannt, dass Andalusien versucht, gesetzlich gegen die Tabakindustrie vorgehen zu können.

\title{
Die Schuldbetreibung
}

\section{Die Fortsetzung der Betreibung}

\section{Fankhauser}

Wie im letzten Artikel [1] dargelegt, kann die Fortsetzung der Betreibung erst verlangt werden, wenn:

- der Schuldner keinen Rechtsvorschlag erhoben hat;

- der Schuldner den Rechtsvorschlag zurückgezogen hat;

- oder wenn der Rechtsvorschlag durch ein Urteil im ordentlichen Verfahren dahingefallen ist.

Sind diese Voraussetzungen gegeben, kann jetzt zum eigentlichen "Vollstreckungsverfahren» geschritten werden.

Korrespondenz:

Margrith Fankhauser

FMH Inkasso Services

Thorackerstrasse 3

CH-3074 Muri b. Bern
Wie das Betreibungsbegehren wird auch das Vollstreckungsverfahren nie von Amtes wegen durchgeführt. Der Gläubiger muss die Fortsetzung der Betreibung ausdrücklich verlangen. Zuständig ist das Betreibungsamt, welches bereits den Zahlungsbefehl erlassen hat. Falls der Schuldner in der Zwischenzeit umgezogen ist, muss das Fortsetzungsbegehren am neuen Wohnsitz des Schuldners eingeleitet werden.

Im Gegensatz zum Einleitungsverfahren hat das Betreibungsamt das Fortsetzungsbegehren zu prüfen, um festzustellen, ob gegen den Zahlungsbefehl Rechtsvorschlag erhoben wurde, dieser zurückgezogen oder beseitigt wurde, und auf welche Art die Betreibung fortgesetzt werden kann. Hier entscheidet sich nämlich, ob die Betreibung auf «Pfändung" oder, wie wir in einem späteren Beitrag aufzeigen werden, auf «Konkurs» weitergeführt werden muss (vgl. Abb. 1).

Wird die Betreibung auf Pfändung fortgesetzt, muss die Pfändung drei Tage nach Eingang des Fortsetzungsbegehrens beim Betreibungsamt vollzogen werden. Der Schuldner muss dem Beamten seine Einkommens- und Vermögenslage offenlegen. Verschweigt er Tatsachen, oder schafft er sogar Vermögenswerte beiseite, kann der Schuldner mit Zuchthaus bis zu fünf Jahren oder Gefängnis bestraft werden. 


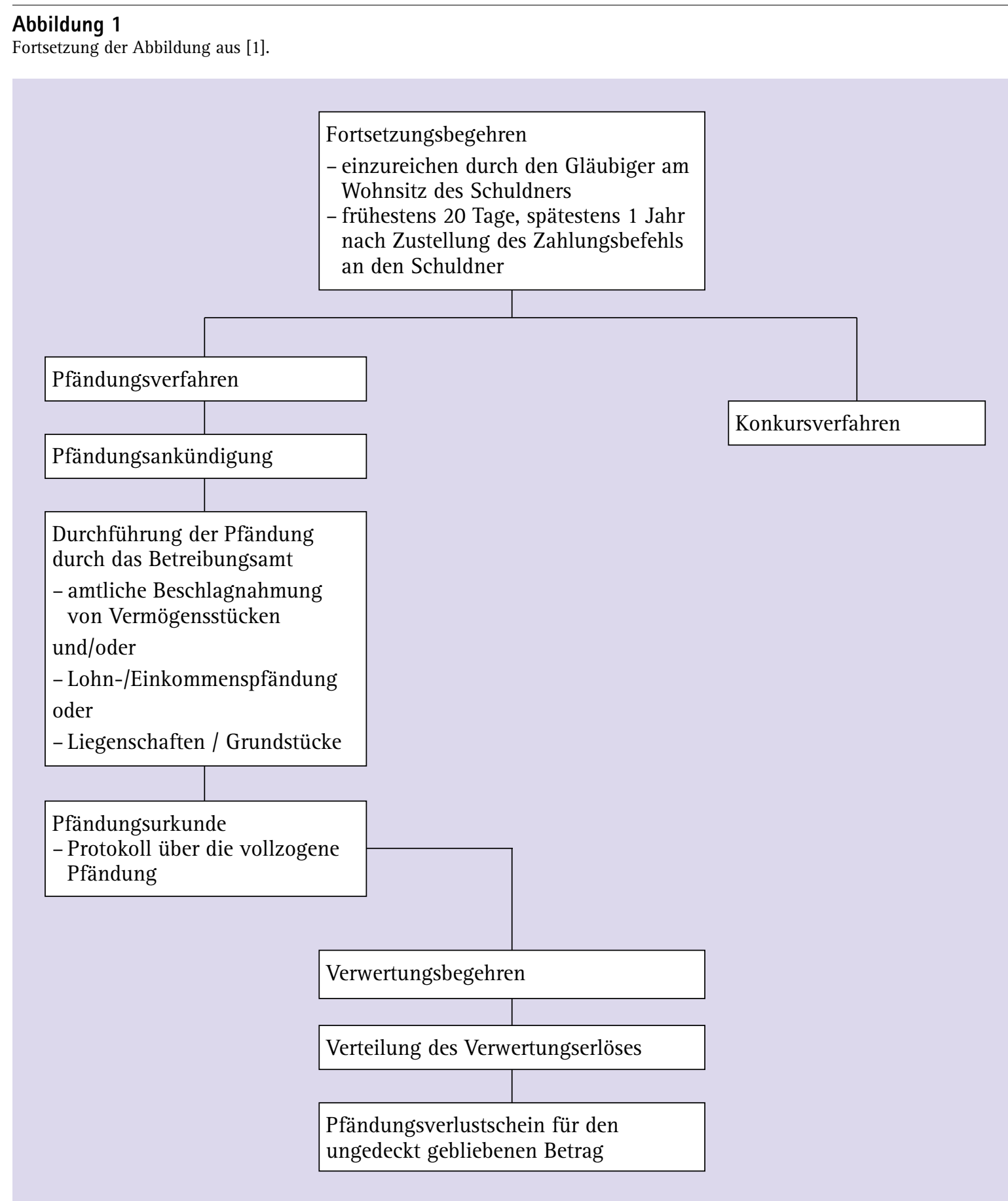

Dem Vollstreckungsziel entsprechend darf nicht mehr gepfändet werden als zur Befriedigung der/des betreibenden Gläubiger/s für ihre/seine Forderungen samt Zins und Kosten benötigt wird. An erster Stelle wird das «bewegliche Vermögen» des Schuldners, Sachen und Forderungen gepfändet. Von der Pfändung ausgenommen sind jedoch die sogenannten «Kompetenzstücke»:

- alle Vermögensstücke, die für den Schuldner und seine Familie zum Leben unentbehrlich sind, wie Kleider, Tisch, Stuhl, Kochgeschirr, Bett usw. sowie
- alle Vermögensstücke, die für den Schuldner und seine Familie zur Ausübung des Berufs unentbehrlich sind, wie Werkzeuge, Geräte, Instrumente, Bücher usw.

Beispiel:

Dem selbständigen Transportunternehmer wird das Fahrzeug als Kompetenzgut belassen, weil er damit sein Einkommen erzielt. Dadurch kann er nicht zuletzt auch seine Schulden tilgen. Mit der Pfändung und der darauf folgenden Verwertung des Fahrzeuges könnte er seine selbständige Tätigkeit nicht 
mehr ausüben. Er müsste sich als Angestellter in einem anderen Unternehmen eine Stelle suchen und könnte dadurch allenfalls ein tieferes Einkommen erzielen oder sogar erwerbslos werden. Sein Notbedarf wäre dann nicht mehr gedeckt und würde nicht ausreichen, um auch den Verpflichtungen seinen Gläubigern gegenüber nachzukommen.

Nicht alle sich in der Wohnung des Schuldners befindlichen Gegenstände können gepfändet werden. Darunter fallen Gegenstände, welche sich zwar beim Schuldner befinden, ihm aber nicht gehören, z.B. ein geleastes Fahrzeug, eine auf Kredit gekaufte Wohnungseinrichtung, ein gemieteter Fernseher, ausgeliehene Gegenstände usw. Häufig werden solche Gegenstände in die Pfändungsurkunde aufgenommen und als Eigentum eines Dritten bezeichnet. Falls Zweifel an den Eigentumsansprüchen des Dritten bestehen, kann der Gläubiger den Anspruch bestreiten und allenfalls Klage erheben (auf eine detailliertere Behandlung dieses Themas verzichten wir aus Gründen der Komplexität).

Liegenschaften und Grundstücke werden nur gepfändet, wenn kein anderes Vermögen vorhanden ist oder wenn der Gläubiger und der Schuldner es gemeinsam verlangen.

An weitaus grösserer Bedeutung gewinnt in der heutigen Zeit jedoch die Erwerbs-, Einkommensresp. Lohnpfändung. Sie ist bequemer, günstiger und in der Regel auch erfolgreicher als die Vermögenspfändung. In etwa 85\% der von uns im letzten Jahr bearbeiteten Inkassofällen, wurden Lohnpfändungen, in nur etwa 15\% Vermögenspfändungen verfügt.

Auch die Einkommens- oder Lohnpfändung unterliegt gesetzlichen Begrenzungen sowohl in zeitlicher wie in "quantitativer» Hinsicht. Es darf nur jener Teil des Lohnes gepfändet werden, der das betreibungsrechtliche Existenzminimum des Schuldners übersteigt. Beschränkt pfändbar sind ausserdem Nutzniessungen und ihre Erträge, Leibrenten sowie Unterhaltsbeiträge, Pensionen und Leistungen jeder
Art, die einen Erwerbsausfall oder Unterhaltsanspruch abgelten (z.B. Invalidenrente).

Unter Existenzminimum (auch Notbedarf genannt) versteht man den Teil des Einkommens, der für den Schuldner und seine Familie unumgänglich notwendig ist. Der Betreibungsbeamte hat das gesetzlich geschützte Existenzminimum nach seinem Ermessen in jedem einzelnen Fall festzusetzen. $\mathrm{Zu}$ bestimmen ist dabei der tatsächliche, objektive Notbedarf des Schuldners und seiner Familie, nicht etwa der standesgemässe oder gewohnte Bedarf! Seitens der kantonalen Aufsichtsbehörden sowie der Konferenz der Betreibungs- und Konkursbeamten in der Schweiz bestehen Richtlinien, die bei der Bemessung berücksichtigt werden müssen. Die Lohnpfändung ist ausserdem zeitlich auf 12 Monate befristet.

Über den Vollzug der Pfändung erstellt das Betreibungsamt die Pfändungsurkunde. Sie bezeichnet den/die Gläubiger und den Schuldner, den Forderungsbetrag, Ort und Zeit der Pfändung und namentlich die einzelnen gepfändeten Vermögensgegenstände mit ihrem Schätzungswert und die allenfalls geltend gemachten Ansprüche Dritter. Wurde (zusätzlich) eine Lohnpfändung verfügt, werden der pfändbare Betrag und die Dauer der Pfändbarkeit festgehalten. Die Pfändungsurkunde ist sozusagen das "vollständige Abbild» aller mit der Pfändung verbundenen Amtshandlungen. Sie ist aber nicht die Pfändung selbst, sondern nur deren urkundliche Feststellung.

Den Abschluss des Pfändungsverfahrens, namentlich das Verwertungsverfahren und die Verteilung des Verwertungserlöses an den/die Gläubiger, werden wir in der Schweizerischen Ärztezeitung Nr. 24 vom 14. Juni 2000 behandeln. Quellenhinweis: WEKA Inkassomanager

\section{Literatur}

1 Fankhauser M. Die Schuldbetreibung. Die Beseitigung des Rechtsvorschlages. Schweiz Ärztezeitung 2000;81(19):987-8.

\section{Korrigendum}

Betrifft Mahler F. Replik. Schweiz Ärztezeitung 2000;81(19):986.

Der korrekte Name des Departementes Herz und Gefässe am Inselspital lautet:

"Schweizer Herz- und Gefässzentrum Bern». 